\title{
Compact Planar Microstripline Branch-Line and Rat-Race Couplers
}

\author{
Kimberley W. Eccleston, Member, IEEE, and Sebastian H. M. Ong, Student Member, IEEE
}

\begin{abstract}
Both branch-line and rat-race couplers are easily realized using planar circuit technology as they employ only transmission lines without additional components. However, as the electrical lengths of the transmission line elements are either $90^{\circ}$ or $270^{\circ}$, such couplers consume a significant amount of circuit area. This paper shows the development of branch-line and rat-race couplers that use artificial transmission lines (ATLs) in place of conventional transmission lines resulting in significant size reduction. As the ATLs are constructed entirely from microstriplines, the couplers are easily fabricated using conventional printed-circuit processes. The design formulas developed for the ATLs are explicit. Full-wave simulation and experimental results were used to confirm the design approach for hybrids operating at $1.8 \mathrm{GHz}$. The frequency response of the proposed hybrids is similar to conventional hybrids.
\end{abstract}

Index Terms-Artificial transmission lines (ATLs), branch-line coupler, periodically loaded transmission lines, rat-race coupler.

\section{INTRODUCTION}

B OTH $90^{\circ}$ and $180^{\circ}$ hybrids find numerous application in microwave systems [1], [2]. In planar microwave integrated circuit technologies, $90^{\circ}$ couplers are often realized using either a branch-line coupler [1]-[4] or Lange coupler [1], [2], [4], [5], while the $180^{\circ}$ coupler is usually realized using a rat-race coupler [1], [2], [4], [6]. The Lange coupler is compact and offers wide bandwidth [5], but requires very narrow and closely spaced interdigitated microstriplines, as well as crossovers; and is best realized using thin-film or monolithic-microwave integrated-circuit (MMIC) technology. On the other hand, branch-line and rat-race hybrids, which employ entire transmission-line elements, are easily realized using a standard single-layer printed-circuit-board fabrication technology.

The branch-line coupler [see Fig. 1(a)] has dimensions of a quarter-wave length by quarter-wavelength at the center frequency, while a rat-race coupler [see Fig. 1(b)] has dimensions a half-wavelength by quarter-wavelength. Due to these large electrical lengths of the transmission-line elements, branch-line and rat-race couplers occupy a significant amount of circuit area. This problem worsens with decreasing frequency. These couplers are often realized in a circular rather than a rectangular

Manuscript received February 6, 2003; revised April 21, 2003.

K. W. Eccleston is with the Department of Electrical and Computer Engineering, National University of Singapore, Singapore 119260, Singapore (e-mail: elekwe@nus.edu.sg).

S. H. M. Ong was with the Department of Electrical and Computer Engineering, National University of Singapore, Singapore 119260, Singapore.

He is now with the Ministry of Education, Singapore 138675, Singapore.

Digital Object Identifier 10.1109/TMTT.2003.817442

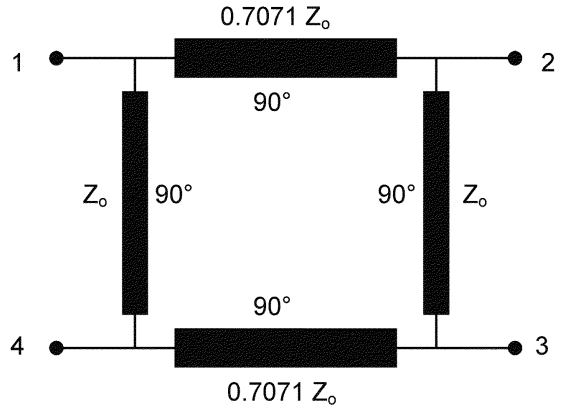

(a)

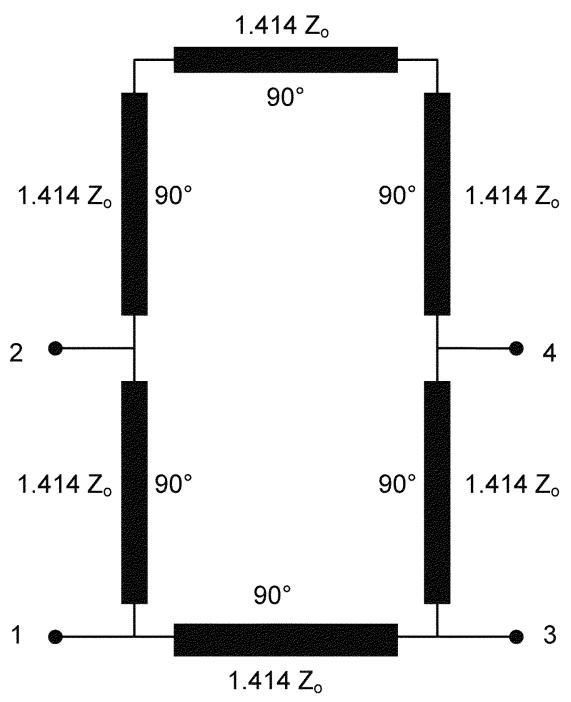

(b)

Fig. 1. Hybrids. (a) Branch-line coupler. (b) Rat-race coupler; where $Z_{o}$ is the system reference impedance.

ring geometry, in which case, even more circuit area is occupied. In either case, the interior of the ring is unoccupied.

Meandering of transmission lines [7] or coupled lines [8] is a well-known method of miniaturization. The level of miniaturization is determined by the number of meander sections and the tightness of the meandering. However, each meander section adds four discontinuities (bends) and, thus, many sections result in many discontinuities (e.g., [9]). Tight meandering results in increased parasitic coupling between transmission-line sections [8]-[10]. This problem is lessened if a thin-film substrate [9], [10] is used.

Lumped elements may be introduced at strategic points [9], [11]-[13] or the coupler completely realized with lumped elements [14]-[17], thereby resulting in significant size reduction; but are more suited to MMIC realization. Certain photonic-bandgap (PBG) structures [18] can be used to shorten the 


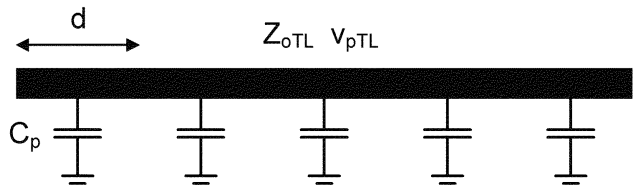

(a)

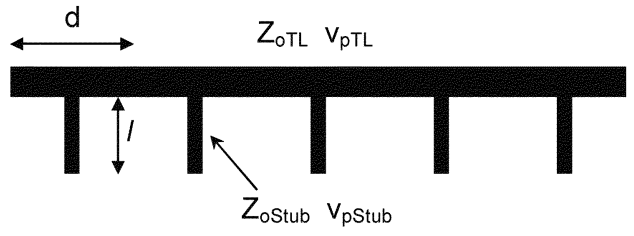

(b)

Fig. 2. ATL assembled from: (a) transmission line and shunt capacitances and (b) transmission line and open-circuit shunt stubs.

microstriplines [19] to result in miniaturization without additional components. However, there are currently no explicit design methods for the PBG elements and a significant area interior to the microstripline ring remains unoccupied. Meandering or spiralling of tightly coupled parallel microstriplines can be used to realize miniature quadrature couplers [9], but requires closely spaced coupled lines.

The artificial transmission line (ATL) concept has been applied to reduce the physical size of planar circuits [20]-[24], of which some methods [22]-[24], however, are more suited to MMICs. In this paper, we present the design of compact hybrids using only transmission-line elements, which can be fabricated with a standard printed-circuit-board etching process and is based upon the ATL concept.

\section{Planar ATLS}

Periodic shunt loading of microstriplines with capacitances can be used to reduce their physical length [20]-[24], as shown in Fig. 2(a). The shunt capacitances are equally spaced, and when the spacing $d$ is much shorter than the guide wavelength, the periodic structure behaves as a transmission line-an ATL - whose effective characteristic impedance $Z_{o \text { ATL }}$ and phase velocity $v_{p \text { ATL }}$ are [25]

$$
\begin{aligned}
& Z_{o \mathrm{ATL}}=\sqrt{\frac{L}{C+\frac{C_{p}}{d}}} \\
& v_{p \mathrm{ATL}}=\frac{1}{\sqrt{L\left(C+\frac{C_{p}}{d}\right)}}
\end{aligned}
$$

where $L$ and $C$ are the distributed inductance and capacitance, respectively, of the transmission-line sections. $L$ and $C$ are related to the transmission-line characteristic impedance $\left(Z_{O \mathrm{TL}}\right)$ and phase velocity $\left(v_{p \mathrm{TL}}\right)$

$$
\begin{aligned}
& Z_{o \mathrm{TL}}=\sqrt{\frac{L}{C}} \\
& v_{p \mathrm{TL}}=\frac{1}{\sqrt{L C}} .
\end{aligned}
$$

Clearly, the effect of periodic capacitive loading is lowered effective characteristic impedance and phase velocity. A lowered

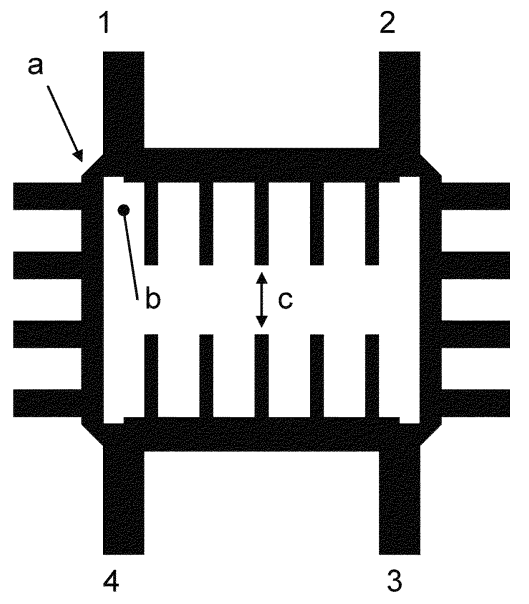

Fig. 3. Proposed microstripline ATL branch-line coupler geometry.

phase velocity means a physically shorter structure can be used to yield a required electrical length compared with a transmission line on its own.

The ATL comprises $N$ (integer) unit cells and, hence, for an $N$ section ATL, its electrical length $\left(\phi_{\text {ATL }}\right)$ is

$$
\phi_{\mathrm{ATL}}=\frac{N d \omega_{o}}{v_{p \mathrm{ATL}}}=N d \omega_{o} \sqrt{L\left(C+\frac{C_{p}}{d}\right)}
$$

where $\omega_{o}$ is the angular frequency of interest. For the case of the hybrids considered in this study, $\phi_{\mathrm{ATL}}$ is either $\pi / 2$ or $\pi$, as the case may be.

$L$ and $C$ can be obtained by solving (3) and (4) and, hence, (1) and (5) can be solved for $d$ and $C_{p}$ for a given transmission line $\left(Z_{o \mathrm{TL}}\right.$ and $\left.v_{p \mathrm{TL}}\right)$ and required values of $Z_{\mathrm{OATL}}$ and $\phi_{\mathrm{ATL}}$ as follows:

$$
\begin{aligned}
d & =\frac{Z_{o \mathrm{ATL}} \phi_{\mathrm{ATL}} v_{p \mathrm{TL}}}{Z_{o \mathrm{TL}} N \omega_{o}} \\
C_{p} & =\frac{\phi_{\mathrm{ATL}}\left(Z_{o \mathrm{TL}}{ }^{2}-Z_{o \mathrm{ATL}}{ }^{2}\right)}{N \omega_{o} Z_{o \mathrm{TL}}{ }^{2} Z_{o \mathrm{ATL}}} .
\end{aligned}
$$

Clearly $Z_{O \mathrm{TL}}$ must be greater than $Z_{O \mathrm{ATL}}$, and the required values of $d$ and $C_{p}$ are inversely proportional to $N$, the number of unit cells. Further, $d$ and, hence, ATL length, is inversely proportional to $Z_{o T L}$. Since the length of the ATL is $N d$, we then see from (6) that the length of the ATL is independent of $N$. Another interesting result is the ratio the ATL length to the length of a transmission line (with phase velocity $v_{p}$ and characteristic impedance $Z_{o \text { ATL }}$, and electrical length $\phi_{\text {ATL }}$ ) is, i.e., $v_{p \mathrm{TL}} Z_{o \mathrm{ATL}} / v_{p} Z_{o \mathrm{TL}}$. (For a given microstripline technology, $v_{p}$ and $v_{p \mathrm{TL}}$ are similar.) This ratio is the level of miniaturization that can be achieved and, clearly, an ATL with low characteristic impedance realized with a high-impedance transmission line will have a short physical length. However, to do this, (7) indicates that $C_{p}$ needs to be large.

The capacitive loading can be realized by shunt stubs, as shown in Fig. 2(b). When realized in microstripline, the resulting structure is completely planar and can be patterned en- 
TABLE I

BRANCH-LINE COUPLER MiCROSTRIPLINE-ELEMENT DiMENSIONS

\begin{tabular}{l|c|c}
\hline & Calculated (mm) & Optimised (mm) \\
\hline $\mathbf{5 0} \mathbf{\Omega} \mathbf{A T L}$ & & \\
$\mathrm{W}_{\mathrm{TL}}$ & 1.30 & 1.30 \\
$\mathrm{~d}$ & 4.19 & 4.20 \\
$\mathrm{~W}_{\text {Stub }}$ & 1.80 & 1.80 \\
$\ell$ & 4.03 & 4.00 \\
$\mathrm{~N}$ & 5 & \\
\hline $\mathbf{3 5} \mathbf{\Omega} \mathbf{A T L}$ & & 1.90 \\
$\mathrm{~W}_{\mathrm{TL}}$ & 1.90 & 3.70 \\
$\mathrm{~d}$ & 3.68 & 1.30 \\
$\mathrm{~W}_{\text {Stub }}$ & 1.30 & 7.80 \\
$\ell$ & 7.77 & \\
$\mathrm{~N}$ & 5 & \\
\hline
\end{tabular}

tirely with one metal layer. The relation between Figs. 2(a) and (b) is

$$
\omega_{o} C_{p}=\frac{1}{Z_{o \text { Stub }}} \tan \left(\frac{\omega_{o}}{v_{p \text { Stub }}} \ell\right) \quad \frac{\omega_{o}}{v_{p \mathrm{Stub}}} \ell<\frac{\pi}{4}
$$

where $Z_{o \text { Stub }}$ and $v_{p \text { Stub }}$ are, respectively, the characteristic impedance and phase velocity of the stub transmission lines. If the lengths of the stubs are short, they behave similar to lumped capacitances and, hence, (8) becomes

$$
C_{p}=\frac{\ell}{Z_{o \text { Stub }} v_{p \text { Stub }}} \quad \frac{\omega_{o}}{v_{p \text { Stub }}} \ell \ll 1
$$

Once the required value of $C_{p}$ is obtained from (7), $\ell$ can be obtained by solving either (8) or (9) for a given stub transmission line $\left(Z_{o \text { Stub }}\right.$ and $\left.v_{p \text { Stub }}\right)$.

\section{Design Considerations AND Method}

The ATLs need to be realizable within the limitations of the technology and have a geometry amenable and beneficial to application in the couplers. Further, the ATLs need to behave in a manner closely resembling that of the transmission lines they are meant to replace. This is achieved when $d$ and $\ell$ are less than one-tenth of the guide wavelength at the highest frequency of interest. Small values of $d$ and $\ell$ translate into short and narrow ATLs, respectively.

In this discussion, we shall confine ourselves to realization in microstripline, noting that other transmission-line technologies may require other considerations and constraints. To minimize the effects of conductor loss, radiation loss, and prevention of spurious modes, the range of the microstripline width is limited and, hence, limiting the range of characteristic impedances that can be realized. Further, it is necessary to have a spacing of three substrate heights $(3 h)$ between elements to minimize crosstalk [1].

One way to reduce stub length is to place $N$ stubs on both sides of the microstripline. The stub lengths are approximately halved, and the bandwidth of the ATL is improved, but the width of the ATL is about the same. We see from (8) or (9) that stub
TABLE II

\begin{tabular}{|c|c|c|}
\hline & Calculated (mm) & Optimised (mm) \\
\hline $\begin{array}{c}70.7 \Omega \text { ATL } \\
\text { (stubs pointed inward) }\end{array}$ & & \\
\hline $\mathrm{W}_{\mathrm{TL}}$ & 0.50 & 0.50 \\
\hline $\mathrm{d}$ & 3.85 & 3.90 \\
\hline $\mathrm{W}_{\text {Stub }}$ & 0.60 & 0.60 \\
\hline$\ell$ & 5.87 & 6.00 \\
\hline $\mathrm{N}$ (per $90^{\circ}$ section) & 5 & \\
\hline $\begin{array}{c}70.7 \Omega \mathrm{ATL} \\
\text { (stubs pointed outward) }\end{array}$ & & \\
\hline $\mathrm{W}_{\mathrm{TL}}$ & 0.50 & 0.50 \\
\hline $\mathrm{d}$ & 3.85 & 3.90 \\
\hline $\mathrm{W}_{\text {Stub }}$ & 1.50 & 1.50 \\
\hline$\ell$ & 3.78 & 3.80 \\
\hline $\mathrm{N}$ & 5 & \\
\hline
\end{tabular}

RAT-RACE COUPLER MiCRostRIPLINE-ELEMENT DiMENSIONS

length $\ell$ (and, hence, ATL width) increases with both $C_{p}$ and $Z_{o \text { Stub }}$. Thus, although increasing $Z_{o \mathrm{TL}}$ reduces the length of the ATL (by reducing $d$ ), it increases $C_{p}$, thereby increasing the ATL width. Equation (7) indicates that $C_{p}$ and, hence, the stub length, may be reduced by increasing $N$. Equations (8) and (9) indicate that the stub length may be reduced by reducing $Z_{o S t u b}$ and, hence, increasing stub width.

However, one needs to note that increasing $N$, increasing stub width, and reducing $d$ reduces the spacing between stubs, thereby causing unwanted coupling between stubs. This problem can be overcome by placing alternate stubs on the other side of the microstripline, but the width of the ATL nearly doubles.

With the above points in mind, the design of a short ATL (and, hence, compact coupler) is, therefore, a constrained optimization problem. It was found that the geometry of the form shown in Fig. 3 results in the best level of miniaturization for the branch-line coupler. The number of stubs on each pair of parallel ATLs may be arbitrary (and not necessarily 5 and 4, as shown). An important attribute of this geometry is that the stubs of two of the ATLs are directed into the interior of the structure, thereby making optimum use of the circuit area. The bend (" $a$ " in Fig. 3), at the ends of the outward directed ATLs ensures that the gap ("b" in Fig. 3) is greater than 3h. A similar geometry may be used for the rat-race coupler.

Based on the above considerations, the general approach used by the authors to design the couplers is as follows. Let $W_{\mathrm{TL}}$ and $W_{\text {Stub }}$ be the widths of the transmission line and stub microstripline, respectively. For a given value of $Z_{O A T L}$ and $N, d$, and $\ell$ (with $W_{\text {Stub }}$ set to $d-3 h$ ) can be calculated for discrete values of $W_{\mathrm{TL}}$. This is repeated for all the required values of $Z_{o \text { ATL }}$. For a pair of identical ATLs (corresponding to a certain value of $W_{\mathrm{TL}}$ ), with inward pointing stubs, the shortest pair of ATLs, with outward pointing stubs, is selected so that the distance (" $c$ " in Fig. 3) between the stub ends of the inward pointing stubs is at least $3 h$. This is repeated for other values of $W_{\mathrm{TL}}$ of the ATLs with inward pointing stubs. The structure with the smallest area can then be chosen. In some cases, it is possible 


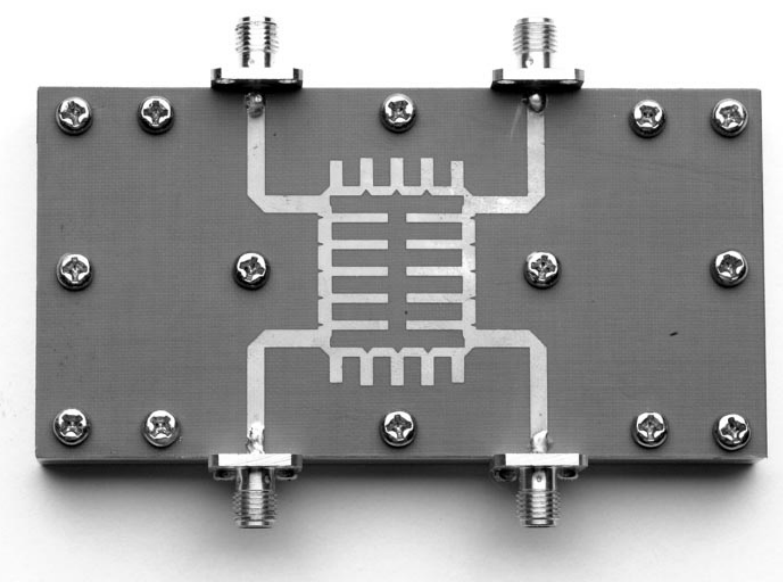

Fig. 4. Photograph of fabricated 1.8-GHz compact branch-line hybrid.

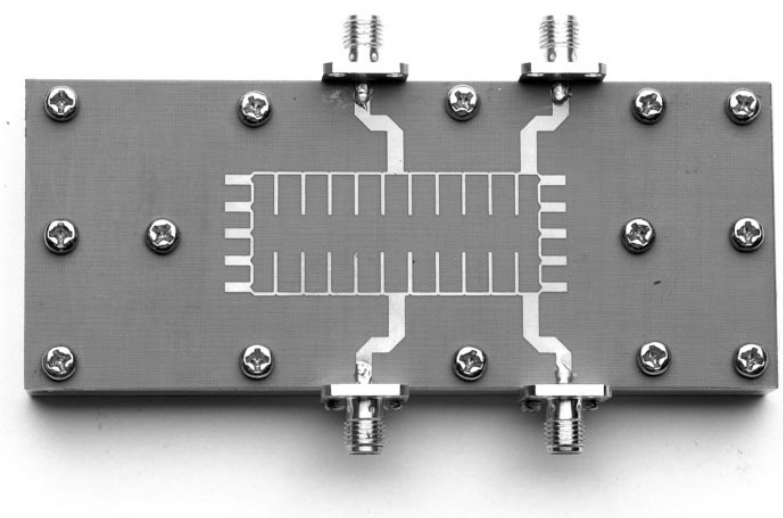

Fig. 5. Photograph of fabricated $1.8-\mathrm{GHz}$ compact rat-race hybrid.

to reduce the widths of the inward pointing stubs, when the distance between the ends of these stubs (" $c$ " in Fig. 3) is greater than $3 h$, thereby reducing coupling between stubs of the same ATL. To ensure acceptable ATL behavior, the lengths of elements need to be checked to ensure that they are less than 0.1 guide wavelength at the highest frequency of interest.

\section{EXPERIMENT AND DISCUSSION}

The above approach was applied to the design of compact hybrids for operation at $1.8 \mathrm{GHz}$. The substrate used has a height of $31 \mathrm{mil}(0.7874 \mathrm{~mm})$ and a dielectric constant of 2.2. The system reference impedance $Z_{o}$ is $50 \Omega$. The smallest microstripline width that could be accurately reproduced with our etching facilities was $0.5 \mathrm{~mm}$. The largest microstripline width considered was $4.5 \mathrm{~mm}$ and corresponds to about $1 / 20$ guide wavelength at $2.5 \mathrm{GHz}$. The optimum design for both the branch-line and rate-race couplers was calculated using the method described in Section III. The stub widths are a considerable proportion of the ATL length $(d)$, mitering [26] of T-junctions and was done to minimize their effects. Mitering [26] was also applied to the bends. Stub lengths can also be foreshortened to compensate for

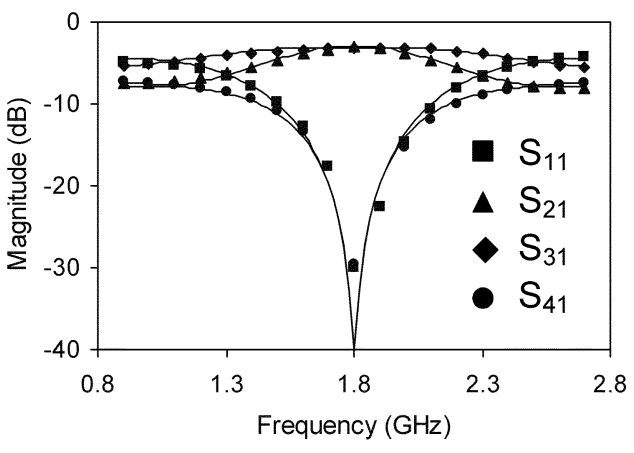

(a)

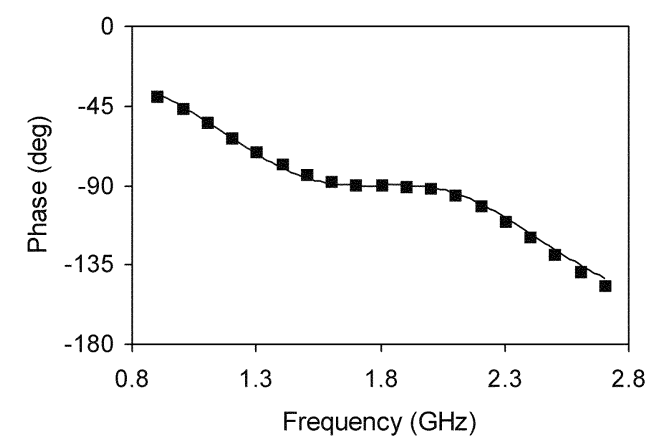

(b)

Fig. 6. Comparison of ideal branch-line coupler (solid lines) and simulated compact branch-line coupler (discrete points). (a) Magnitudes of $S_{11}, S_{21}, S_{31}$, and $S_{41}$. (b) Phase of $S_{31} / S_{21}$.

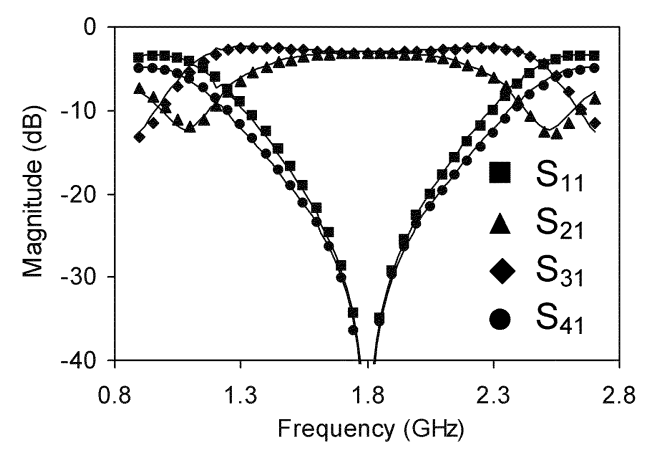

(a)

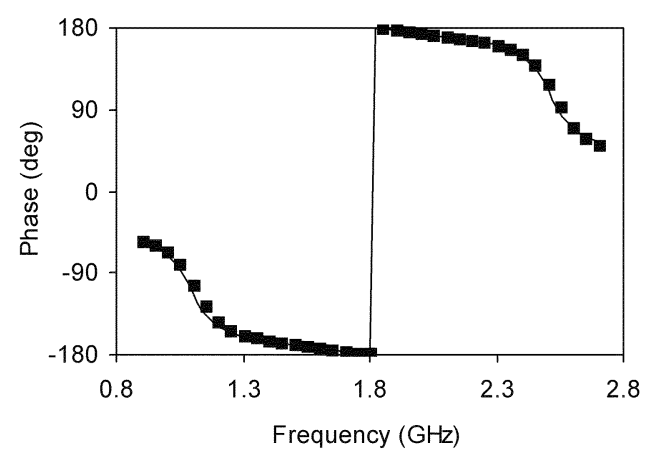

(b)

Fig. 7. Comparison of ideal rat-race coupler (solid lines) and simulated compact rat-race coupler (discrete points). (a) Magnitudes of $S_{11}, S_{21}, S_{31}$, and $S_{41}$. (b) Phase of $S_{42} / S_{43}$. 


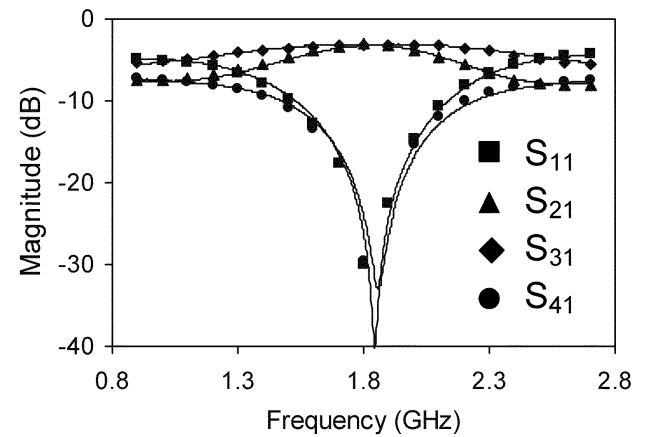

(a)

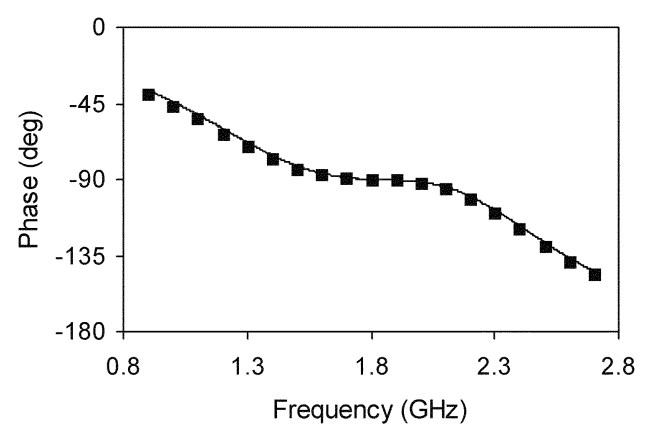

(b)

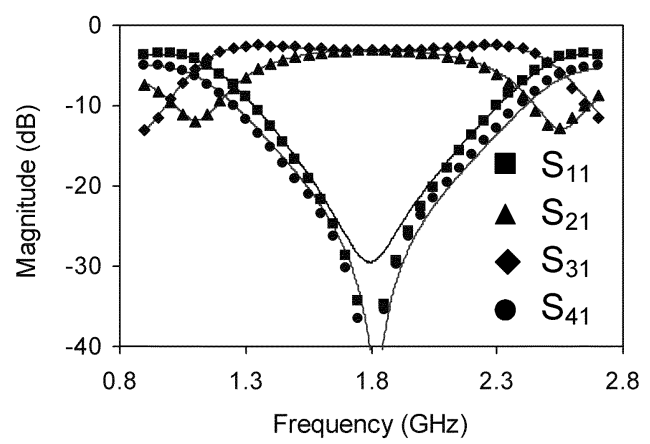

(a)

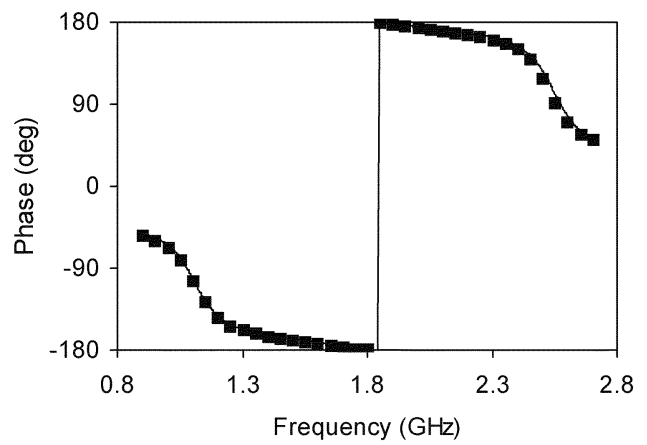

(b)
Fig. 8. Comparison of measurements (solid lines) and simulations (discrete points) for the branch-line coupler. (a) Magnitudes of $S_{11}, S_{21}, S_{31}$, and $S_{41}$. (b) Phase of $S_{31} / S_{21}$.

the end fringing. However, in this project, due to the low-frequency and low dielectric constant of the substrate, the level of compensation is very small and was not considered.

To account for the effects of microstripline discontinuities and coupling between microstripline elements, Sonnet ${ }^{1}$ -which can perform full-wave electromagnetic (EM) analysis of planar circuits-was used to simulate the circuit layout. In this package, the sidewalls are restricted to perfect conductors, while there is flexibility for choice of the top boundary. The grid spacing was set to $0.1 \mathrm{~mm}$ and is sufficiently small for the frequency range under consideration. Conductor and dielectric losses were considered in the simulations. The cover height was set to $10 \mathrm{~mm}$ above the substrate surface, but simulations performed without a cover (free-space boundary condition) did not reveal a significant difference, indicating that radiation loss is minimal.

In the first part of the simulations, the ATLs were simulated on their own to establish that they emulate transmission-line behavior at least over the passband of the hybrid and had the expected effective ATL characteristic impedance. The entire hybrid circuit layout was then simulated to confirm hybrid behavior, account for the addition of microstripline ports, and facilitate fine tuning of the circuit layout. Tables I and II show the calculated and fine-tuned circuit element dimensions for the 1.8-GHz branch-line and rate-race couplers, respectively.

${ }^{1}$ Sonnet LIte 8.51, Sonnet Software Inc., Liverpool, NY. [Online]. Available: http://www.sonnetusa.com
Fig. 9. Comparison of measurements (solid lines) and simulations (discrete points) for the rat-race coupler. (a) Magnitudes of $S_{11}, S_{21}, S_{31}$ and $S_{41}$ (b) Phase of $S_{42} / S_{43}$.

Only minimal adjustment of microstripline elements was necessary and is a result of the constraints imposed in the design calculations and compensation of discontinuities. Figs. 4 and 5 are photographs of the fabricated branch-line and rat-race couplers, respectively.

Figs. 6 and 7 compare the simulated frequency response of representative $S$-parameters (port mismatch, coupled, and isolated port transmission) of the compact couplers with that of couplers constructed from ideal transmission lines. The differences in behavior are virtually indistinguishable apart from an insertion loss of approximately $0.1 \mathrm{~dB}$ and an in-balance of approximately $0.1 \mathrm{~dB}$ at the center frequency. Figs. 6 and 7, therefore, illustrate the validity of design principles. This outcome is a consequence of the constraints placed on the microstripline-element lengths, thereby ensuring that the structures behave as transmission lines over at least the passband of the coupler. Figs. 8 and 9 compare the measured and simulated frequency responses of representative $S$-parameters of the compact couplers. The measurements were made using an HP8510 vector network analyzer. Figs. 8 and 9 show that the measured and simulated $S$-parameters agree very well, and this outcome is expected for full-wave simulation at this frequency range. The main difference between the measurements and simulations is a slight shift of center frequency (of less than 3\%) of both the port match and isolation for both couplers. 
TABLE III

COMPARISON OF CIRCUIT AREA OF THE 1.8-GHz COUPLERS

\begin{tabular}{l|l|l|l}
\hline & \multicolumn{3}{|c}{ Area $\left(\mathrm{mm}^{2}\right)$} \\
\hline & $\begin{array}{l}\text { Conventional } \\
\text { Rectangular } \\
\text { Geometry }\end{array}$ & $\begin{array}{l}\text { Conventional } \\
\text { Circular } \\
\text { Geometry }\end{array}$ & $\begin{array}{l}\text { Proposed } \\
\text { Structure }\end{array}$ \\
\hline Branch-line & 1088 & 1412 & 687 \\
\hline Rat-race & 2016 & 2846 & 922 \\
\hline
\end{tabular}

Table III shows a comparison of the circuit areas occupied by conventional coupler designs and that proposed in this paper. The size of the proposed branch-line coupler is between $49 \%-63 \%$ of a conventional design, while the size of the proposed rat-race coupler is between $32 \%-46 \%$ of a conventional design. This level of miniaturization is expected to be achievable for other center frequencies and substrates.

\section{CONCLUSION}

In this paper, we have shown the development and design method for compact branch-line and rat-race couplers that can be realized in microstripline with one layer of metal without additional lumped elements. The miniaturization is achieved by using ATLs consisting of microstriplines periodically loaded with open-circuit shunt stubs in place of transmission lines. The design method uses explicit formulas and considers technology constraints and the area occupied by the circuit. The method was demonstrated on the realization of couplers operating at $1.8 \mathrm{GHz}$ by way of both full-wave simulations, as well as measurements of prototypes. The size of the proposed couplers compared to conventional designs is between $49 \%-63 \%$, and $32 \%-46 \%$ for the branch-line and rat-race couplers, respectively. The element sizes of the components can easily be patterned using standard printed-circuit etching processes. The proposed couplers have frequency responses (and, hence, bandwidths) similar to conventional couplers. At these frequencies, conductor and dielectric losses were found to be insignificant. Simulations suggest that radiation loss is also insignificant.

\section{ACKNOWLEDGMENT}

The authors wish to express gratitude to the following technical staff from the National University of Singapore, Singapore: S. C. Lee, and L. H. Chan for their assistance in fabricating the microstripline circuits, and C. H. Sing for measuring the $S$-parameters of the circuits.

\section{REFERENCES}

[1] I. Bahl and P. Bhartia, Microwave Solid-State Circuit Design. New York: Wiley, 1988.
[2] S. A. Maas, Nonlinear Microwave Circuits. Piscataway, NJ: IEEE Press, 1997.

[3] C. G. Montgomery, R. H. Dicke, and E. M. Purcell, Principles of Microwave Circuits. New York: McGraw-Hill, 1948.

[4] D. M. Pozar, Microwave Engineering, 2nd ed. New York: Wiley, 1998.

[5] J. Lange, "Interdigitated stripline quadrature hybrid," IEEE Trans. Microwave Theory Tech., vol. MTT-17, pp. 1150-1151, Dec. 1969.

[6] W. A. Tyrell, "Hybrid circuits for microwaves," Proc. IRE, vol. 35, pp. 1294-1306, Nov. 1947

[7] E. G. Cristal, "Meander-line and hybrid meander-line transformers," IEEE Trans. Microwave Theory Tech., vol. MTT-21, pp. 69-76, Feb. 1973.

[8] H. Tanaka, Y. Sasaki, T. Hashimoto, Y. Yagi, and Y. Ishikawa, "Miniaturized 90 degree hybrid coupler using high dielectric substrate for QPSK modulator," in IEEE MTT-S Int. Microwave Symp. Dig., 1996, pp. 793-796.

[9] I. Toyoda, T. Hirota, T. Hiraoka, and T. Tokumitsu, "Multilayer MMIC brach-line coupler and broad-side coupler," in $\mathrm{Mi}$ crowave Millimeter-Wave Monolithic Circuits Symp. Dig., 1992, pp. $79-82$.

[10] T. Hasegawa, S. Banba, and H. Ogawa, "A branchline hybrid using valley microstrip lines," IEEE Microwave Guided Wave Lett., vol. 2, pp. 76-78, Feb. 1992.

[11] D. P. Andrews and C. S. Aitchison, "Wide-band lumped element quadrature 3-dB couplers in microstrip," IEEE Trans. Microwave Theory Tech., vol. 48, pp. 2424-2431, Dec. 2000.

[12] T. Hirota, A. Minakawa, and M. Muraguchi, "Reduced-size branch-line and rat-race hybrids for uniplanar MMICs," IEEE Trans. Microwave Theory Tech., vol. 38, pp. 270-275, Mar. 1990.

[13] M. C. Scardelletti, G. E. Ponchak, and T. M. Weller, "Miniaturized Wilkinson power dividers utilizing capacitive loading," IEEE Microwave Wireless Comp. Lett., vol. 12, pp. 6-8, Jan. 2002.

[14] H. R. Ahn, I. S. Chang, and S. W. Yun, "Miniaturized 3-dB ring hybrid terminated by arbitrary impedances," IEEE Trans. Microwave Theory Tech., vol. 42, pp. 2216-2221, Dec. 1994.

[15] F. Giannini and L. Scucchia, "A double frequency $180^{\circ}$ lumped-element hybrid," Microwave Optical Technol. Lett., vol. 33, no. 4, pp. 247-251, May 2002.

[16] Y. C. Chiang and C. Y. Chen, "Design of a wide-band lumped-element 3-dB quadrature coupler," IEEE Trans. Microwave Theory Tech., vol. 49, pp. 476-479, Mar. 2001.

[17] T. Kawai, Y. Kokubo, and I. Ohta, "Broadband lumped-element 180degree hybrids utilizing lattice circuits," in IEEE MTT-S Int. Microwave Symp. Dig., 2001, pp. 47-50.

[18] Q. Xue, K. M. Shum, and C. H. Chan, "Novel 1-D microstrip PBG cells," IEEE Microwave Guided Wave Lett., vol. 10, pp. 403-405, Oct. 2000

[19] K. M. Shum, Q. Xue, and C. H. Chan, "A novel microstrip ring hybrid incorporating a PBG cell," IEEE Microwave Wireless Comp. Lett., vol. 11, pp. 258-260, June 2001.

[20] J. S. Hong and M. J. Lancaster, "Capacitively loaded microstrip loop resonator," Electron. Lett., vol. 30, no. 18, pp. 1494-1495, Sept. 1994.

[21] A. Görür, C. Karpuz, and M. Alkan, "Characteristics of periodically loaded CPW structures," IEEE Microwave Guided Wave Lett., vol. 8, pp. 278-280, Aug. 1998.

[22] P. Kangaslahti, P. Alinikula, and V. Porra, "Monolithic artificial transmission line balanced frequency doublers," in Eur. Microwave Conf., 1997, pp. 219-224.

[23] — , "Miniaturized artificial-transmission-line monolithic millimeter-wave frequency doubler," IEEE Trans. Microwave Theory Tech., vol. 48, pp. 510-518, Apr. 2000.

[24] H. T. Kim, J. H. Park, Y. K. Kim, and Y. Kwon, "Compact low-loss monolithic CPW filters using air-gap overlay structures," IEEE Microwave Wireless Comp. Lett., vol. 11, pp. 328-330, Aug. 2001.

[25] R. E. Collin, Foundations for Microwave Engineering. New York: McGraw-Hill, 1992, ch. 8.

[26] R. Chadha and K. C. Gupta, "Compensation of discontinuities in planar transmission lines," IEEE Trans. Microwave Theory Tech., vol. MTT-30, pp. 2151-2156, Dec. 1982. 


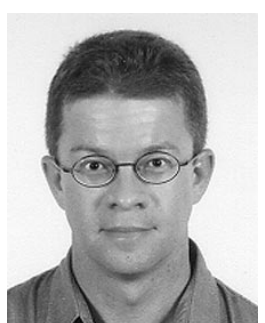

Kimberley W. Eccleston (S'85-M'90) was born in Melbourne, Australia, in 1964. He received the BE degree in electronic engineering (with distinction) from the Royal Melbourne Institute of Technology (RMIT), Melbourne, Australia, in 1986, and the $\mathrm{Ph} . \mathrm{D}$. degree from the University of Queensland, Brisbane, Australia, in 1991.

From 1990 to 1992, he was a Research Scientist with the Defence Science and Technology Organization, Adelaide, Australia. Since 1992, he has been with the Department of Electrical and Computer Engineering, National University of Singapore (NUS), Singapore, where he is currently a Senior Lecturer. He has taught students at various levels and has developed specialized courses in microwave active circuit design at both the undergraduate and graduate levels. He has authored over 35 technical papers He holds one patent. He possesses experience in microwave measurements and microwave device modeling. His research is currently focused on active and passive microwave circuit design.

Dr. Eccleston has been a member of organizing committees of several IEEE conferences hosted in Singapore.

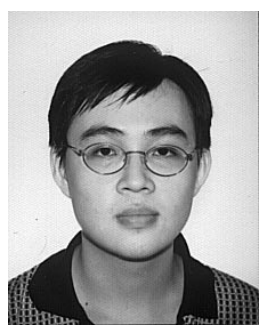

Sebastian H. M. Ong (S'03) was born in Singapore, in 1978. He received the B.Eng. degree in electrical engineering (with honors) from the National University of Singapore, Singapore, in 2003. His final year project was on the design of compact microstripline hybrids.

Upon graduation, he joined the Ministry of Education, Singapore. 